ELECTRONIC RESEARCH ANNOUNCEMENTS OF THE AMERICAN MATHEMATICAL SOCIETY

Volume 6, Pages 64-74 (September 11, 2000)

S $1079-6762(00) 00082-2$

\title{
LOCAL DIMENSIONS FOR POINCARÉ RECURRENCES
}

\author{
VALENTIN AFRAIMOVICH, JEAN-RENÉ CHAZOTTES, AND BENOÎT SAUSSOL
}

(Communicated by Svetlana Katok)

\begin{abstract}
Pointwise dimensions and spectra for measures associated with Poincaré recurrences are calculated for arbitrary weakly specified subshifts with positive entropy and for the corresponding special flows. It is proved that the Poincaré recurrence for a "typical" cylinder is asymptotically its length. Examples are provided which show that this is not true for some systems with zero entropy. Precise formulas for dimensions of measures associated with Poincaré recurrences are derived, which are comparable to Young's formula for the Hausdorff dimension of measures and Abramov's formula for the entropy of special flows.
\end{abstract}

\section{INTRODUCTION}

Poincaré recurrences are main indicators and characteristics of the repetition of behavior of dynamical systems in time. A traditional approach is to study statistical properties of the quantity $\tau_{U}(x)$, the first return time of the orbit through $x$ into a set $U$. We adopt another point of view: instead of looking at the mean return time or at the return time of points, we are going to study $\tau(U)$, the smallest possible return time into $U$, that is we define the Poincaré recurrence for a set, as the infimum over all return times of the points inside the set 1]. Poincaré recurrences for a set $U$ can be very different from return times $\tau_{U}(x)$. If $U=\xi^{n}(x)$ is a cylinder of length $n$, then the return time $\tau_{\xi^{n}(x)}(x)$ of a $\mu$-generic point behaves like $\exp \left(n \mathrm{~h}_{\mu}(T, \xi)\right)$ [7] (where $T$ is the map generating the dynamical system, and $\mathrm{h}_{\mu}(T, \xi)$ is the entropy of $\mu$, with respect to $T$ and $\xi$ ), whereas the Poincaré recurrence for $\xi^{n}(x)$ is typically of order $n$, provided that $\mu$ is ergodic, $T$ is weakly specified and $\mathrm{h}_{\mu}(T, \xi)>0$ (Theorem 2 below). Let us emphasize that this result does not depend on a particular choice of a map $T$, a partition $\xi$ and an ergodic measure $\mu$. Since we deal with a function of sets (namely $U \mapsto \tau(U)$ ), it is natural to use ideas and methods of dimension theory [9]. We define and calculate pointwise dimensions for Poincaré recurrences (Theorems 4 and 6) to obtain spectra for measures (Theorems [5] and 7). These quantities reflect the balance between times needed for the return to the ball $B(x, \varepsilon)$ (of radius $\varepsilon$ centered at $x$ ) and $\varepsilon$ for almost every point $x$ with respect to an arbitrary ergodic measure, provided that $\varepsilon$ is small enough. This provides a new insight into the nature of recurrences. We remark that the positivity of the entropy is an unavoidable assumption (Theorem 3 ).

This work is part of the manuscript 2 .

Received by the editors March 31, 2000 and, in revised form, May 8, 2000.

2000 Mathematics Subject Classification. Primary 37C45, 37B20.

(C)2000 American Mathematical Society 


\section{SETUP FOR MAPS}

In this work we shall deal with dynamical systems $(X, T)$ which are weakly specified subshifts. This means that there exists a finite set $\Sigma$, called the alphabet, and $X$ is a closed subset of $\Sigma^{\mathbb{N}}$ (non-invertible case) or $\Sigma^{\mathbb{Z}}$ (invertible case) which is invariant under the shift map $T$ defined by $(T x)_{i}=x_{i+1}$. Given $a \in \Sigma$ let $[a]=\left\{x \in X: x_{0}=a\right\}$, and let $\xi=\{[a]: a \in \Sigma\}$ denote the partition into 1-cylinders. We endow $X$ with the product topology, which makes $X$ a compact metrizable space. Our results concern only measures with positive entropy, so we will assume that $(X, T)$ has positive topological entropy. We also assume that $(X, T)$ is weakly specified; see [6]. We now define a metric on $X$ equivalent to the product topology (see Lemma 1).

Case when $T$ is not invertible. Denote by $\xi^{n}$ the dynamical partition, that is: $\xi^{n} \stackrel{\text { def }}{=}$ $\bigvee_{j=0}^{n-1} T^{-j} \xi, \xi^{0} \stackrel{\text { def }}{=}\{X, \emptyset\}$. Then $\xi^{n}(x)$ will be the atom of the refined partition $\xi^{n}$ that contains $x$ and will be referred to as the $n$-cylinder about $x$. Given a continuous function $\mathrm{u}: X \rightarrow(0, \infty)$ we endow $X$ with the metric $\mathrm{d}_{X}$ defined by $\mathrm{d}_{X}(x, y) \stackrel{\text { def }}{=} \mathrm{e}^{-\mathrm{u}\left(\xi^{n}(x)\right)}$ whenever $y \in \xi^{n}(x)$ and $y \notin \xi^{n+1}(x)$, where

$$
\mathrm{u}\left(\xi^{n}(x)\right)=\sup _{k \leq n} \sup _{z \in \xi^{k}(x)}\left(\mathrm{u}(z)+\mathrm{u}(T z)+\cdots+\mathrm{u}\left(T^{k-1} z\right)\right), n=1,2, \ldots .
$$

Remark that the standard metric is recovered when one chooses $\mathrm{u} \equiv 1$. If one chooses $\mathrm{u}(x)=-\log \lambda\left(x_{0}\right)$, which is a constant on every atom of $\xi$, then

$$
\mathrm{d}_{X}(x, y)=\prod_{\ell=0}^{n-1} \lambda\left(x_{\ell}\right), \quad \text { and } \quad \operatorname{diam} \xi^{n}(x)=\prod_{\ell=0}^{n-1} \lambda\left(x_{\ell}\right),
$$

i.e. we have a situation similar to that encountered in Moran-like geometric construction. More generally, if one chooses a Hölder continuous function $\mathrm{u}$, then one gets the distance used to generate Cantor-like sets in $\mathbb{R}^{d}[9,3]$ modeled by subshifts.

Case when $T$ is invertible. Denote by $\xi_{m}^{n}$ the dynamical partition, that is: $\xi_{m}^{n} \stackrel{\text { def }}{=}$ $T^{m} \xi \vee T^{m-1} \xi \vee \cdots \vee T^{-n+1} \xi, \xi_{0}^{0} \stackrel{\text { def }}{=}\{X, \emptyset\}$, where $m \geq 0, n \geq 0$. Then $\xi_{m}^{n}(x)$ will be the atom of the refined partition $\xi_{m}^{n}$ that contains $x$ and will be referred to as the $(m, n)$-cylinder about $x$. Given two continuous functions $\mathrm{u}, \mathrm{v}: X \rightarrow(0, \infty)$ such that $\mathrm{u}(x)=\mathrm{u}(y)$ whenever $\xi_{0}^{n}(x)=\xi_{0}^{n}(y)$ for every $n \geq 0$ and $\mathrm{v}(x)=\mathrm{v}(y)$ whenever $\xi_{m}^{0}(x)=\xi_{m}^{0}(y)$ for every $m \geq 0$, we endow $X$ with the metric $\mathrm{d}_{X}$ defined by (11) below. For an arbitrary pair $x, y \in X$, there is a unique pair $(m, n)$ such that $y \in \xi_{m}^{n}(x)$ and $y \notin\left(\xi_{m+1}^{n}(x) \cup \xi_{m}^{n+1}(x)\right)$. Then

$$
\mathrm{d}_{X}(x, y) \stackrel{\text { def }}{=} \max \left\{\mathrm{e}^{-\mathrm{u}\left(\xi_{0}^{n}(x)\right)}, \mathrm{e}^{-\mathrm{v}\left(\xi_{m}^{0}(x)\right)}\right\},
$$

where

$$
\begin{gathered}
\mathrm{u}\left(\xi_{0}^{n}(x)\right)=\sup _{k \leq n} \sup _{z \in \xi_{0}^{k}(x)}\left(\mathrm{u}(z)+\mathrm{u}(T z)+\cdots+\mathrm{u}\left(T^{k-1} z\right)\right), n=1,2, \ldots, \\
\mathrm{v}\left(\xi_{m}^{0}(x)\right)=\sup _{k \leq m} \sup _{z \in \xi_{k}^{0}(x)}\left(\mathrm{v}(z)+\mathrm{v}\left(T^{-1} z\right)+\cdots+\mathrm{v}\left(T^{-k+1} z\right)\right), m=1,2, \ldots .
\end{gathered}
$$


If one chooses $\mathrm{u}(x)=-\log \lambda\left(x_{0}\right), \mathrm{v}(x)=-\log \gamma\left(x_{-1}\right)$, which are constants on every atom of $\xi_{0}^{1}$ and $\xi_{1}^{0}$, respectively, then

$$
\operatorname{diam} \xi_{m}^{n}(x)=\max \left\{\prod_{\ell=1}^{m} \gamma\left(x_{-\ell}\right), \prod_{\ell=0}^{n-1} \lambda\left(x_{\ell}\right)\right\} .
$$

Such a situation occurs, for example, in the case of a piecewise linear Smale horseshoe. In the general case of basic axiom A sets on surfaces, there exist an associated subshift of finite type $(X, T)$ and some functions $\mathrm{u}, \mathrm{v}$ satisfying the above assumptions (i.e. depending only on forward, respectively backward, itineraries) giving rise to a metric $\mathrm{d}_{X}$ which is "adapted" to the initial system.

Given $x \in X$ and $\varepsilon \geq 0$ we denote by $B(x, \varepsilon)$ the open ball of radius $\varepsilon$ centered at $x$. The proof of the following lemma will be omitted.

Lemma 1. $\left(X, \mathrm{~d}_{X}\right)$ is an ultra-metric space, and for any $x \in X$ and $\varepsilon>0$ we have

1. $B(x, \varepsilon)=\xi^{n_{x, \varepsilon}}(x)$, where we set $n_{x, \varepsilon}=\min \left\{n \in \mathbb{N}: \mathrm{e}^{-\mathrm{u}\left(\xi^{n}(x)\right)}<\varepsilon\right\}$ in the non-invertible case.

2. $B(x, \varepsilon)=\xi_{m_{x, \varepsilon}}^{n_{x, \varepsilon}}(x)$, where we set $n_{x, \varepsilon}=\min \left\{n \in \mathbb{N}: \mathrm{e}^{-\mathrm{u}\left(\xi_{0}^{n}(x)\right)}<\varepsilon\right\}$ and $m_{x, \varepsilon}=\min \left\{m \in \mathbb{N}: \mathrm{e}^{-\mathrm{u}\left(\xi_{0}^{m}(x)\right)}<\varepsilon\right\}$ in the invertible case.

3. The topology generated by $d$ is equivalent to the product topology.

\section{PoincARÉ RECURRENCES OF SETS AND LOCAL DIMENSIONS}

For any set $U \subset X$, one can define the first return time of a point $x \in U$ into $U$ :

$$
\tau_{U}(x) \stackrel{\text { def }}{=} \inf \left\{k \geq 1: T^{k} x \in U\right\} .
$$

By convention we put this return time to be infinite if the point $x$ never comes back to $U$.

Definition 1 ([1]). Let $U$ be a subset of $X$. Then,

$$
\tau_{T}(U) \stackrel{\text { def }}{=} \inf \left\{\tau_{U}(x): x \in U\right\}
$$

In the following lemma we collect various basic properties of the Poincaré recurrence of sets. The proof will be omitted.

Lemma 2. Let $(X, T)$ be a dynamical system and $U \subset X$ any set. Then the following properties hold:

1. $\tau_{T}(U)=\inf \left\{k>0: T^{k} U \cap U \neq \emptyset\right\}=\inf \left\{k>0: T^{-k} U \cap U \neq \emptyset\right\}$.

2. $\tau_{T}(U)=\tau_{T}\left(T^{-1} U\right)$. If $T$ is invertible, then $\tau_{T}(T U)=\tau_{T}(U)$.

3. Monotonicity: $A \subset B \Rightarrow \tau_{T}(A) \geq \tau_{T}(B)$.

Spectra for Poincaré recurrences. For any $A \subset X$, any $\alpha \in \mathbb{R}$ and any $q \in \mathbb{R}$, we define

$$
\mathcal{M}^{T}(A, \alpha, q, \varepsilon) \stackrel{\text { def }}{=} \inf _{\substack{\left(x_{i}, \varepsilon_{i}\right) \\ \varepsilon_{i} \leq \varepsilon}} \sum_{i} \exp \left(-q \tau_{T}\left(B\left(x_{i}, \varepsilon_{i}\right)\right)\right) \varepsilon_{i}^{\alpha},
$$

where the infimum is taken over all finite or countable collections $\left(x_{i}, \varepsilon_{i}\right)$ such that $\bigcup_{i} B\left(x_{i}, \varepsilon_{i}\right) \supseteq A$. The limit $\mathcal{M}^{T}(A, \alpha, q) \stackrel{\text { def }}{=} \lim _{\varepsilon \rightarrow 0} \mathcal{M}^{T}(A, \alpha, q, \varepsilon)$ exists by monotonicity and we give the following definition (which was first stated in [1]): 
Definition 2. For any non-empty $A \subset X$ and any $q \in \mathbb{R}$,

$$
\alpha_{T}(A, q) \stackrel{\text { def }}{=} \begin{cases}\inf \left\{\alpha: \mathcal{M}^{T}(A, \alpha, q)=0\right\} & \text { if } q \geq 0, \\ \sup \left\{\alpha: \mathcal{M}^{T}(A, \alpha, q)=\infty\right\} & \text { if } q<0,\end{cases}
$$

is called the spectrum for Poincaré recurrences for the map $T$ of the set $A$.

It is easy to see that whenever $\alpha_{T}(A, q)$ is finite one has

$$
\alpha_{T}(A, q)=\inf \left\{\alpha: \mathcal{M}^{T}(A, \alpha, q)=0\right\}=\sup \left\{\alpha: \mathcal{M}^{T}(A, \alpha, q)=\infty\right\} .
$$

Note that $\alpha_{T}(A, 0)$ is nothing but the Hausdorff dimension of the set $A$. Since spectra for Poincaré recurrences are just Carathéodory dimensions for corresponding Carathéodory structures, we may define, following [9], the following quantity.

Definition 3. Let $\alpha_{T}(\cdot, q)$ be the spectrum defined above. Let $\mu$ be a Borel probability measure on $X$. Then

$$
\alpha_{T}^{\mu}(q) \stackrel{\text { def }}{=} \inf \left\{\alpha_{T}(Y, q): Y \subset X, \mu(Y)=1\right\} .
$$

We will call it the spectrum for the measure $\mu$.

We emphasize that the family of sets used to define (21) (here, balls) is very important to get non-trivial results for the spectrum of the measure. A similar quantity (for $\alpha=0$ ) is studied in [8], using covers by open sets in (2). Basically it is proven therein that the spectrum of an invariant ergodic measure is always trivial.

Pointwise dimensions for Poincaré recurrences. Following the ideas of [9], we define the following quantities.

Definition 4 (Lower and upper pointwise dimensions). The lower and upper pointwise dimensions of $\mu$ at a point $x$ are defined by

$$
\underline{\bar{d}}_{\mu, q}^{T}(x) \stackrel{\text { def }}{=} \varlimsup_{\varepsilon \rightarrow 0} \frac{\log \mu(B(x, \varepsilon))+q \tau_{T}(B(x, \varepsilon))}{\log \varepsilon} .
$$

This definition is not exactly as in 9 . However, by adopting such a definition we may show directly that spectrum for a measure coincides with this quantity for almost every point $x$ (Theorems 4 and 5 ). The relationship between Definition 4 and Pesin's definition of pointwise dimension 9] was studied in [5] in the case of the general Carathéodory construction.

\section{MAIN RESULTS FOR MAPS}

To find formulas for pointwise dimensions and to establish their coincidence with the spectrum for a measure, we need to know Poincaré recurrences for "typical" cylinders.

\section{Local rate of Poincaré recurrences for cylinders.}

Definition 5. Lower and upper local rates of Poincaré recurrences for cylinders are defined respectively for non-invertible and invertible transformations by

$$
\underline{\overline{\mathcal{R}}}_{\xi}(x) \stackrel{\text { def }}{=} \varlimsup_{n \rightarrow \infty} \frac{\tau_{T}\left(\xi^{n}(x)\right)}{n} \text { and } \quad \overline{\mathcal{R}}_{\xi}(x) \stackrel{\text { def }}{=} \varlimsup_{n+m \rightarrow \infty} \frac{\tau_{T}\left(\xi_{m}^{n}(x)\right)}{m+n} .
$$

Weak specification property (see [6]) immediately implies the following result (we omit the proof): 
Proposition 1. If the system $(X, T)$ is weakly specified, then $\overline{\mathcal{R}}_{\xi}(x) \leq 1$.

The following result established in the non-invertible case in [10], by using ideas Kolmogorov's complexity, will be crucial in what follows. We shall give a direct proof based on the Shannon-McMillan-Breiman theorem.

Theorem 1. Let $(X, \mathfrak{B}, \mu)$ be a probability space where $\mu$ is ergodic with respect to a measurable transformation $T: X \rightarrow X$. If $\xi$ is a finite or countable measurable partition with strictly positive entropy $h_{\mu}(T, \xi)$, then the lower rate of Poincaré recurrences for cylinders is almost surely bigger than one:

$$
\underline{\mathcal{R}}_{\xi}(x) \geq 1 \text { for } \mu \text {-a.e. } x \in X .
$$

Coming back to our setup and putting together Proposition 1 and Theorem 1 we obtain the following theorem:

Theorem 2. Let $\mu$ be an ergodic measure of positive entropy on the weakly specified subshift $(X, T)$, and $\xi$ the finite partition of $X$ defined in the setup. Then

$$
\underline{\mathcal{R}}_{\xi}(x)=\overline{\mathcal{R}}_{\xi}(x) \stackrel{\text { def }}{=} \mathcal{R}_{\xi}(x)=1 \quad \text { for } \mu \text {-a.e. } x \in X .
$$

The following examples show that for systems with zero entropy the statement of Theorem 1 and a fortiori the one of Theorem 2$]$ may not hold.

Examples in the case of zero entropy systems. A first example is given by the dyadic adding machine. In this case, it is simple to show that $\mathcal{R} \equiv 0$.

We now consider a more interesting situation, namely a rotation $f_{\omega}: x \mapsto$ $x-\omega \bmod 1$ (i.e. $f_{\omega}^{-1} x=x+\omega \bmod 1$ ), on the circle $\mathcal{S}^{1}=\mathbb{R} / \mathbb{Z}$, where $0<\omega<1$ is an irrational number. The number $\omega$ can be approximated by rational numbers $p / q$ ( $p$ and $q$ are relatively prime) in such a way that

$$
\left|\omega-\frac{p}{q}\right|<\frac{1}{q^{\beta+1}}
$$

for some value $\beta$ and some pair $(p, q)$. Let $\beta(\omega) \stackrel{\text { def }}{=} \sup \beta$, where the supremum is taken over all $\beta$ for which inequality (5) has infinitely many solutions $(p, q)$ with $q>0$. Assume that $\beta(\omega)<\infty$, i.e. $\omega$ is a Diophantine number. Then for every $\delta \in(0,1)$ the inequality

$$
\left|\omega-\frac{p}{q}\right|<\frac{1}{q^{\beta(\omega)+1-\delta}}
$$

holds for infinitely many relatively prime pairs $\left(p_{i}, q_{i}\right)$, with $q_{i} \rightarrow \infty$ as $i \rightarrow \infty$. Consider the partition $\xi$ of $\mathcal{S}^{1}$ made up of two intervals $[0, \omega)$ and $[\omega, 1)$. The rotation is metrically isomorphic to the subshift $\operatorname{clos}\left(\pi \mathcal{S}^{1}\right)$, where the coding map $\pi: \mathcal{S}^{1} \rightarrow\{0,1\}^{\mathbb{N}}$ is defined in the obvious way by $\pi(x)_{n}=0$ if $f_{\omega}^{n}(x) \in[0, \omega)$ and $\pi(x)_{n}=1$ if $f_{\omega}^{n}(x) \in[\omega, 1)$.

We now state the following theorem:

Theorem 3. If $\beta(\omega)>3$ then

$$
\underline{\mathcal{R}}_{\xi}(x)=0
$$

for almost every $x$ with respect to Lebesgue measure on $\mathcal{S}^{1}$. 
Existence of pointwise dimensions and formulas of spectra. One can find formulas for pointwise dimensions and spectra for measures.

Theorem 4. Under the assumptions of Theorem $\mathbb{Q}$, for any $q \in \mathbb{R}$ and for $\mu$-a.e. $x \in X$, one has:

$$
\begin{gathered}
\underline{d}_{\mu, q}^{T}(x)=\bar{d}_{\mu, q}^{T}(x)=\frac{\mathrm{h}_{\mu}(T)-q}{\int \mathrm{u} d \mu} \quad \text { in the non-invertible case, and } \\
\underline{d}_{\mu, q}^{T}(x)=\bar{d}_{\mu, q}^{T}(x)=\left(\mathrm{h}_{\mu}(T)-q\right)\left(\frac{1}{\int \mathrm{u} d \mu}+\frac{1}{\int \mathrm{v} d \mu}\right) \quad \text { in the invertible case. }
\end{gathered}
$$

From this result, we can deduce the expression for the spectrum for the measure:

Theorem 5. Under the assumptions of Theorem 2 , for any $q \in \mathbb{R}$ one has

$$
\begin{gathered}
\alpha_{T}^{\mu}(q)=\frac{\mathrm{h}_{\mu}(T)-q}{\int \mathrm{u} d \mu} \quad \text { in the non-invertible case, and } \\
\alpha_{T}^{\mu}(q)=\left(\mathrm{h}_{\mu}(T)-q\right)\left(\frac{1}{\int \mathrm{u} d \mu}+\frac{1}{\int \mathrm{v} d \mu}\right) \quad \text { in the invertible case. }
\end{gathered}
$$

One could see these relations as an analog of Young's formula [11] for dimensions for Poincaré recurrences. It is explicit if $q=0$. Note also that the spectrum for the set $X, \alpha(X, q)$, was obtained for certain subshifts in [3], where it has been shown that it satisfies a non-homogeneous Bowen's equation.

Corollary 1. The value of $q$ for which $\alpha_{T}^{\mu}$ vanishes is equal to $h_{\mu}(T) \stackrel{\text { def }}{=} q_{0}^{T}$.

\section{SETUP FOR SPECIAL FLOWS}

Let $\varphi$ be a strictly positive continuous function, and $(X, T)$ a compact metric space with a distance $d_{X}$.

Define the special space and the special flow as follows:

$$
X^{\varphi} \stackrel{\text { def }}{=}\{(x, t): x \in X, 0 \leq t \leq \varphi(x)\},
$$

where we identify the points $(x, \varphi(x))$ and $(T x, 0)$ for each $x \in X$, and

$$
\left\{\begin{array}{l}
\Phi_{s}(x, t)=(x, t+s) \text { if } t+s<\varphi(x) \\
\Phi_{s}(x, t)=(T x, t+s-\varphi(x)) \text { if } t+s \geq \varphi(x) .
\end{array}\right.
$$

Assume for a moment that $\varphi(x) \equiv 1$. Let us recall the definition of the distance on $X^{1}$ [4]. Consider the subset $X \times\{t\}$ of $X \times[0,1]$ and let $\rho_{t}$ denote the metric on $X \times\{t\}$ defined by $\rho_{t}((x, t),(y, t)) \stackrel{\text { def }}{=}(1-t) \mathrm{d}_{X}(x, y)+t \mathrm{~d}_{X}(T x, T y), x, y \in X$. Thus, $\rho_{0}((x, 0),(y, 0))=\mathrm{d}_{X}(x, y)$ and $\rho_{1}((x, 1),(y, 1))=\mathrm{d}_{X}(T x, T y)$. Consider a chain $x=w_{0}, w_{1}, \ldots, w_{n}=y$ between $x$ and $y$ where for each $i$ either $w_{i}$ and $w_{i+1}$ belong to $X \times\{t\}$ for some $t\left(\left[w_{i}, w_{i+1}\right]\right.$ is said to be a horizontal segment and length $\left.\left(\left[w_{i}, w_{i+1}\right]\right) \stackrel{\text { def }}{=} \rho_{t}\left(\left(w_{i}, t\right),\left(w_{i+1}, t\right)\right)\right)$ or $w_{i}$ and $w_{i+1}$ are on the same orbit $\left(\left[w_{i}, w_{i+1}\right]\right.$ is said to be a vertical segment and length $\left(\left[w_{i}, w_{i+1}\right]\right)$ is the shortest temporal distance between $w_{i}$ and $w_{i+1}$ along the orbit). The length of the chain is defined to be the sum of the lengths of its segments. Then $d_{X^{1}}((x, s),(y, t))$ is defined to be the infimum of the lengths of all finite chains between $(x, s)$ and $(y, t)$.

We now show that $X^{\varphi}$ and $X^{1}$ are homeomorphic provided that $\varphi(x)>0, x \in$ $X$. Indeed, set $h(x, s) \stackrel{\text { def }}{=}(x, s \varphi(x))$ for any $0 \leq s<1$ and $(x, s) \in X^{1}$ and $h(x, 1)=$ 
$(x, \varphi(x))$. This map is continuous and one-to-one $\left(h^{-1}(x, t)=(x, t / \varphi(x))\right)$. Now, introduce the following distance on $X^{\varphi}$ :

$$
d_{X^{\varphi}}((x, s),(y, t)) \stackrel{\text { def }}{=} d_{X^{1}}\left(h^{-1}(x, s), h^{-1}(y, t)\right) .
$$

Given $(x, s) \in X^{1}, \varepsilon>0$, let

$$
\mathcal{S}((x, s), \varepsilon) \stackrel{\text { def }}{=}\left\{(y, s) \in X^{1}: \rho_{s}((x, s),(y, s))<\frac{\varepsilon}{2}\right\}
$$

and

$$
\begin{array}{r}
\tilde{B}((x, s), \varepsilon) \stackrel{\text { def }}{=}\left\{(y, t) \in X^{1}:|t-s|<\frac{\varepsilon}{2}, \Phi_{s-t}(y, t) \in \mathcal{S}((x, s), \varepsilon), \text { if } s \geq t,\right. \text { and } \\
\left.(y, t)=\Phi_{t-s}\left(y^{\prime}, s\right),\left(y^{\prime}, s\right) \in \mathcal{S}((x, s), \varepsilon), \text { if } t>s\right\},
\end{array}
$$

i.e. $\tilde{B}((x, s), \varepsilon)$ is the set of pieces of temporal length $\varepsilon$ of (semi-)orbits that pass through the "horizontal ball" $\mathcal{S}((x, s), \varepsilon)$. It is clear that if $\tilde{B}((x, s), \varepsilon) \cap X \times(\{0\} \cup$ $\{1\})=\emptyset$, then $\tilde{B}((x, s), \varepsilon)$ is homeomorphic to the direct product of $\mathcal{S}((x, s), \varepsilon)$ and an interval.

Set $B((x, s), \varepsilon) \stackrel{\text { def }}{=} h^{-1} \tilde{B}((x, s), \varepsilon)$. We shall use $B$-sets to cover $X^{\varphi}$; see below.

If $\mu$ is a $T$-invariant probability measure on $X$, then we define a $\Phi$-invariant probability measure $\bar{\mu} \stackrel{\text { def }}{=} \bar{\mu}_{\varphi}$ by putting, for any $F$ continuous on $X^{\varphi}$ :

$$
\int_{X^{\varphi}} F d \bar{\mu} \stackrel{\text { def }}{=} \frac{\int_{X}\left(\int_{0}^{\varphi(x)} F(x, t) d t\right) d \mu(x)}{\int_{X} \varphi(x) d \mu(x)} .
$$

That is, $\bar{\mu}$ is the normalization on $X^{\varphi}$ obtained by taking the direct product of $\mu$ with Lebesgue measure on $\mathbb{R}$. (It can be proved that every $\Phi$-invariant probability measure on $X^{\varphi}$ can be obtained in this way from a $T$-invariant probability measure on $X$ by Fubini's theorem.)

We can now define the first return time of the set $B((x, t), \varepsilon)$ :

Definition 6. For any $x \in X, t>0$ and $\varepsilon>0$, the Poincaré recurrence for the set $B((x, t), \varepsilon)$ is

$$
\tau_{\Phi}(B((x, t), \varepsilon)) \stackrel{\text { def }}{=} \inf \left\{s>\varepsilon: \Phi_{s}(B((x, t), \varepsilon)) \cap B((x, t), \varepsilon) \neq \emptyset\right\} .
$$

Spectra for Poincaré recurrences. For each $Z \subset X^{\varphi}$, each $\alpha \in \mathbb{R}$ and each $q \in \mathbb{R}$, we set

$$
\mathcal{M}^{\Phi}(Z, \alpha, q, \varepsilon) \stackrel{\text { def }}{=} \inf _{\substack{\left(x_{i}, t_{i}, \varepsilon_{i}\right) \\ \varepsilon_{i} \leq \varepsilon}} \sum_{i} \exp \left(-q \tau_{\Phi}\left(B\left(\left(x_{i}, t_{i}\right), \varepsilon_{i}\right)\right)\right) \varepsilon_{i}^{\alpha},
$$

where $B\left(\left(x_{i}, t_{i}\right), \varepsilon_{i}\right)$ is defined above and $\bigcup_{i} B\left(\left(x_{i}, t_{i}\right), \varepsilon_{i}\right) \supseteq Z$. Then the limit $\mathcal{M}^{\Phi}(Z, \alpha, q) \stackrel{\text { def }}{=} \lim _{\varepsilon \rightarrow 0} \mathcal{M}^{\Phi}(Z, \alpha, q, \varepsilon)$ exists by monotonicity and we introduce the following definition:

Definition 7 (Spectrum for flows). For any $Z \subset X^{\varphi}$ and any $q \geq 0$,

$$
\alpha_{\Phi}(Z, q) \stackrel{\text { def }}{=} \sup \left\{\alpha: \mathcal{M}^{\Phi}(Z, \alpha, q)=\infty\right\}
$$

is called the spectrum for Poincaré recurrence for the flow $\Phi$ of the set $Z$.

The quantity $\alpha_{\Phi}(Z, 0)$ coincides with the Hausdorff dimension of $Z$ for the flow. Now we proceed to the definition of the spectra for measures. 
Definition 8 (Spectra for measures). Let $\alpha_{\Phi}(\cdot, q)$ be the spectra defined above. Let $\bar{\mu}$ be a Borel probability measure on $X^{\varphi}$. Then define the following spectrum:

$$
\alpha_{\Phi}^{\bar{\mu}}(q) \stackrel{\text { def }}{=} \inf \left\{\alpha_{\Phi}(V, q): V \in X^{\varphi}, \bar{\mu}(V)=1\right\} .
$$

\section{Pointwise dimensions for Poincaré recurrences.}

Definition 9 (Lower and upper $\Phi$-pointwise dimensions). The lower and upper $\Phi$ pointwise dimensions of $\bar{\mu}$ at the point $(x, t)$ are defined by

$$
\underline{\bar{d}}_{\bar{\mu}, q}^{g \Phi}(x, t) \stackrel{\text { def }}{=} \varliminf_{\varepsilon \rightarrow 0} \frac{\log \bar{\mu}\left(B((x, t), \varepsilon)+q \tau_{\Phi}(B((x, t), \varepsilon))\right.}{\log \varepsilon} .
$$

MAIN RESUlTS FOR SPECIAL FLOWS

We shall call a $B$-set $B((x, s), \varepsilon)$ a "good" one if $B((x, s), \varepsilon)=h^{-1} \tilde{B}((x, s), \varepsilon)$ and there exists a cylinder $\xi_{n}(y)$ such that $\mathcal{S}((x, s), \varepsilon)=\Phi_{s}\left(\xi_{n}(x)\right)$. In this case, we will denote $\xi_{n}(y)$ by $\eta_{n}(B((x, s), \varepsilon))$. Let us denote by $n_{\varepsilon}$ the index $n$ for the cylinder $\eta_{n}(B((x, s), \varepsilon))$, where $B((x, s), \varepsilon)$ is a good $B$-set. For convenience, we shall write $\eta_{n_{\varepsilon}}(x)$ instead of $\eta_{n_{\varepsilon}}(B(x, s), \varepsilon)$ ). Then, the asymptotic behavior of the Poincaré recurrence $\tau_{\Phi}(B((x, t), \varepsilon))$ can be described as follows.

Proposition 2. For $\bar{\mu}$-almost every point $(x, s) \in X^{\varphi}$,

$$
\lim _{\varepsilon \rightarrow 0} \frac{\tau_{\Phi}((B(x, s), \varepsilon))}{n_{\varepsilon}}=\int \varphi d \mu .
$$

By using this proposition, we prove the following results.

Theorem 6. Let $\bar{\mu}$ be the measure on $X^{\varphi}$ induced by the ergodic measure $\mu$ on $X$. The pointwise dimension exists $\bar{\mu}$-a.e. and is equal to

$$
\begin{gathered}
d_{\bar{\mu}, q}^{\Phi}(x, t)=1+\frac{1}{\int \mathrm{u} d \mu}\left(\mathrm{h}_{\mu}(T)-q \int \varphi d \mu\right) \text { in the non-invertible case, and } \\
d_{\frac{\Phi}{\mu}, q}^{\Phi}(x, t)=1+\left(\frac{1}{\int \mathrm{u} d \mu}+\frac{1}{\int \mathrm{v} d \mu}\right)\left(\mathrm{h}_{\mu}(T)-q \int \varphi d \mu\right) \text { in the invertible case. }
\end{gathered}
$$

Now we can state the following theorem:

Theorem 7. The spectrum for the measure $\bar{\mu}$ is given for all $q \in \mathbb{R}$ by

$$
\begin{gathered}
\alpha_{\Phi}^{\bar{\mu}}(q)=1+\frac{1}{\int \mathrm{u} d \mu}\left(\mathrm{h}_{\mu}(T)-q \int \varphi d \mu\right) \text { in the non-invertible case, and } \\
\alpha_{\Phi}^{\bar{\mu}}(q)=1+\left(\frac{1}{\int \mathrm{u} d \mu}+\frac{1}{\int \mathrm{v} d \mu}\right)\left(\mathrm{h}_{\mu}(T)-q \int \varphi d \mu\right) \text { in the invertible case. }
\end{gathered}
$$

Corollary 2. We have

$$
\alpha_{\Phi}^{\bar{\mu}}\left(q / \int \varphi d \mu\right)=1+\alpha_{T}^{\mu}(q) .
$$

The value of $q$ for which $\alpha_{\Phi}^{\bar{\mu}}$ vanishes is equal to

$$
\begin{gathered}
\frac{\mathrm{h}_{\mu}(T)+\int \mathrm{u} d \mu}{\int \varphi d \mu} \stackrel{\text { def }}{=} q_{0}^{\Phi}=\frac{q_{0}^{T}+\int \mathrm{u} d \mu}{\int \varphi d \mu} \text { in the non-invertible case, and } \\
\frac{\mathrm{h}_{\mu}(T)+\frac{\int \mathrm{u} d \mu \times \int \mathrm{v} d \mu}{\int(\mathrm{u}+\mathrm{v}) d \mu}}{\int \varphi d \mu} \stackrel{\text { def }}{=} q_{0}^{\Phi}=\frac{q_{0}^{T}+\frac{\int \mathrm{u} d \mu \times \int \mathrm{v} d \mu}{\int(\mathrm{u}+\mathrm{v}) d \mu}}{\int \varphi d \mu} \text { in the invertible case. }
\end{gathered}
$$




\section{Proof of Theorem 1}

For the sake of definiteness, we write the proof for the case of invertible $T$. The case of non-invertible $T$ can be obtained in a similar way after evident simplifications.

It suffices to prove the theorem for finite partitions; the case of countable $\xi$ will follow easily. More precisely, if $\xi=\left\{B_{1}, B_{2}, \ldots\right\}$ is countable, then for some $m<\infty$ the finite partition $\hat{\xi}=\left\{B_{1}, B_{2}, \ldots, B_{m}, \bigcup_{l>m} B_{l}\right\}$ will have positive entropy. In addition, $\xi$ is finer than $\hat{\xi}$, hence $\tau_{T}\left(\xi_{m}^{n}(x)\right) \geq \tau_{T}\left(\hat{\xi}_{m}^{n}(x)\right)$, and the statement follows.

Assume now that $\xi$ is finite. Observe that $h \stackrel{\text { def }}{=} h_{\mu}(T, \xi)$ is non-zero and finite. Fix $\varepsilon \in(0, h / 3)$. By the Shannon-McMillan-Breiman theorem for $\mu$-a.e. $x$, there exists $N(x)$ such that if $n+m>N(x)$ then

$$
\left|\frac{1}{n+m} \log \mu\left(\xi_{m}^{n}(x)\right)+h\right| \leq \varepsilon
$$

By Egoroff's theorem, if $M=M(\varepsilon)$ is sufficiently large then $E_{M} \stackrel{\text { def }}{=}\{x \in X$ : $N(x)<M\}$ will have a measure $\mu\left(E_{M(\varepsilon)}\right)>1-\varepsilon$. We can choose $c$ so large that for any $x \in E_{M(\varepsilon)}$ and any positive integers $n, m$

$$
c^{-1} e^{[-(n+m) h-(n+m) \varepsilon]} \leq \mu\left(\xi_{m}^{n}(x)\right) \leq c e^{[-(n+m) h+(n+m) \varepsilon]} .
$$

We now write $E=E_{M(\varepsilon)}$. Let $\delta=1-\frac{3}{h} \varepsilon$ and set

$$
\mathcal{A}_{m}^{n} \stackrel{\text { def }}{=}\left\{x \in E: \tau_{T}\left(\xi_{m}^{n}(x)\right) \leq \delta(n+m)\right\} .
$$

Obviously $\mathcal{A}_{m}^{n}=\bigcup_{k=1}^{\delta(n+m)} R_{m}^{n}(k)$ where

$$
R_{m}^{n}(k) \stackrel{\text { def }}{=}\left\{x \in E: \tau_{T}\left(\xi_{m}^{n}(x)\right)=k\right\} .
$$

We shall prove that $\sum_{n, m} \mu\left(\mathcal{A}_{m}^{n}\right)<\infty$. Let $n, m$ be positive integers and $0 \leq k \leq$ $n+m$. If the return time of the cylinder $C=\left[a_{-m} a_{-m+1} \cdots a_{0} \cdots a_{n-1}\right] \in \xi_{m}^{n}$ is equal to $k$, i.e. $\tau_{T}(C)=k$, then it can be readily checked that $a_{j+k}=a_{j}$, for all $-m \leq j \leq n-k-1$. This means that any block made with $k$ consecutive symbols completely determines the cylinder $C$. In particular, since there exists $p \geq 0$ such that $p \leq m$ and $0 \leq k-p \leq n$, we can choose the cylinder $Z=\xi_{p}^{k-p}(x) \supset \xi_{m}^{n}(x)$. Let

$$
\mathcal{Z}=\left\{\xi_{p}^{k-p}(x): x \in R_{m}^{n}(k)\right\} .
$$

Because of the structure of cylinders under consideration, for any cylinder $Z \in \mathcal{Z}$ there exists a (unique) cylinder $C_{Z} \in \xi_{m}^{n}$ such that $C_{Z} \subset Z$ and $Z \cap R_{m}^{n}(k) \subset C_{Z}$. This implies

$$
\mu\left(R_{m}^{n}(k)\right)=\sum_{Z \in \mathcal{Z}} \mu\left(Z \cap R_{m}^{n}(k)\right) \leq \sum_{Z \in \mathcal{Z}} \mu\left(C_{Z}\right) .
$$

By definition, for each $Z \in \mathcal{Z}$, there exists $x \in E$ such that $Z=\xi_{p}^{k-p}(x)$ and $C_{Z}=\xi_{m}^{n}(x)$. Using (15) we get

$$
\mu\left(\xi_{m}^{n}(x)\right) \leq c \mathrm{e}^{[-(n+m) h+(n+m) \varepsilon]} \quad \text { and } \quad 1 \leq c \mu\left(\xi_{p}^{k-p}(x)\right) \mathrm{e}^{[k h+k \varepsilon]} .
$$

Multiplying these inequalities we get

$$
\mu\left(C_{Z}\right) \leq c^{2} \exp [-(n+m) h+(n+m) \varepsilon] \exp [k h+k \varepsilon] \mu(Z) .
$$


Summing up on $Z \in \mathcal{Z}$ we get (recall that $k \leq n+m$ )

$$
\mu\left(R_{m}^{n}(k)\right) \leq c^{2} \exp [-(n+m-k) h+2(n+m) \varepsilon] .
$$

This implies that

$$
\begin{aligned}
\mu\left(\mathcal{A}_{m}^{n}\right) & =\sum_{k=1}^{[\delta(n+m)]} \mu\left(R_{m}^{n}(k)\right) \\
& \leq c^{2} \frac{e^{h}}{e^{h}-1} \exp [-(n+m)(h-\delta h-2 \varepsilon)] .
\end{aligned}
$$

Since $h-\delta h-2 \varepsilon=h-\left(1-\frac{3}{h} \varepsilon\right) h-2 \varepsilon=\varepsilon>0$, we get that

$$
\sum_{m \geq 1, n \geq 1} \mu\left(\mathcal{A}_{m}^{n}\right)<+\infty .
$$

In view of the Borel-Cantelli lemma, we finally get that for $\mu$-almost every $x \in E$, $\tau_{T}\left(\xi_{m}^{n}(x)\right) \geq\left(1-\frac{3}{h} \varepsilon\right)(n+m)$ except for finitely many pairs of integers $(n, m)$. Since in addition $\mu(E)>1-\varepsilon$, the arbitrariness of $\varepsilon$ implies the desired result

\section{ACKNOWLEDGMENTS}

V. A. is supported by CONACYT through its program of "Cátedras Patrimoniales II" under contract 485100-2. J.-R. C. and B. S. are grateful to the Instituto de Investigación en Comunicación Optica UASLP for hospitality during their visit to San Luis Potosi. B. S. is supported by FCT's Funding Program and by the Center for Mathematical Analysis, Geometry, and Dynamical Systems.

\section{REFERENCES}

[1] V. Afraimovich, Pesin's dimension for Poincaré recurrences, Chaos 7 (1997), 12-20. MR 98c:58080

[2] V. Afraimovich, J.-R. Chazottes, and B. Saussol, Pointwise dimensions for Poincaré recurrences associated with maps and special flows, in preparation.

[3] V. Afraimovich, J. Schmeling, E. Ugalde, and J. Urías, Spectra of dimensions for Poincaré recurrences, to appear in Discrete and Continuous Dynamical Systems (2000).

[4] R. Bowen and P. Walters, Expansive one-parameter flows, J. Diff. Equas. 12 (1972), 180-193. MR 49:6202

[5] J.-R. Chazottes and B. Saussol, Sur les dimensions locales et les dimensions des mesures, preprint (2000)

[6] A. Katok and B. Hasselblatt, Introduction to the modern theory of dynamical systems, Encyclopedia of Math. and its Applications 54, Cambridge University Press, Cambridge, 1995. MR 96c:58055

[7] D. S. Ornstein and B. Weiss, Entropy and data compression schemes, IEEE Transactions on Information Theory 39 (1993), 78-83. MR 93m:94012

[8] V. Penné, B. Saussol, and S. Vaienti, Dimensions for recurrence times: topological and dynamical properties, Discrete and Continuous Dynamical Systems 5 (1999), 783-798. MR 2000j:37026

[9] Ya. B. Pesin, Dimension theory in dynamical systems, contemporary views and applications, Chicago Lectures in Mathematics, 1997. MR 99b:58003

[10] B. Saussol, S. Troubetzkoy, and S. Vaienti, In preparation.

[11] L.-S. Young, Dimension, entropy and Lyapunov exponents, Ergod. Th. \& Dyn. Syst 2 (1982), 109-124. MR 84h:58087 
74 VALENTIN AFRAIMOVICH, JEAN-RENÉ CHAZOTTES, AND BENOÎT SAUSSOL

IICO-UASLP, A. Obregon 64, San Luis Potosi SLP, 78210 MeXico

E-mail address: valentin@cactus.iico.uaslp.mx

IICO-UASLP, A. Obregon 64, San Luis Potosi SLP, 78210 Mexico

E-mail address: jeanrene@cpt.univ-mrs.fr

Departamento de Matemática, Instituto Superior Técnico, 1049-001 Lisboa, Portugal

E-mail address: saussol@math.ist.utl.pt 